\title{
POTENCIALIDADES DAS ATIVIDADES DE TELECONSULTORIAS EM NUTRIÇÃO
}

\author{
Potentialities of Teleconsulting in Nutrition
}

\section{Cintia Chaves Curioni'; Maria Thereza Furtado Cury²; Michelle Guimarães do Carmo ${ }^{3}$}

Resumo Objetivos: Apresentar os resultados e potencialidades das atividades de teleconsultoria realizadas pelo núcleo Telenutrição-RJ. Materiais e métodos: Dados obtidos através de relatórios administrativos gerados pelo sistema Moodle desde o início das atividades do núcleo, em 2009. Resultados: Foram realizadas 17 teleconsultorias no período de abril de 2012 a setembro de 2013. As solicitações foram feitas prioritariamente por nutricionistas (70\%). Apesar Teleconsultorias serem focadas para o Estado do Rio de Janeiro (35\% das solicitações) observa-se uma grande demanda de outros estados, principalmente Minas Gerais (35\%) e São Paulo (12\%). Conclusão: A teleconsultoria é uma nova forma de trabalho, porém ainda pouco inserida no cotidiano dos profissionais da atenção primária de saúde. São necessárias estratégias que promovam um maior vínculo entre profissionais de saúde e equipe do Telenutrição-RJ, auxiliando na melhor utilização da ferramenta.

Palavras-chave: Telessaúde, Telenutrição, Atenção Primária de Saúde, Teleconsultoria

Aims: This paper presents the results and potentialities of teleconsultation in Nutrition performed by the Telenutrition from the Telehealth-RJ. Materials and methods: Data were obtained by administrative reports generated by "Moodle system" since the beginning of the activities. Results: From April 2012 to September 2013, 17 teleconsultations were requested by different practitioners,

Abstract of whom 70\% were nutritionists. Despite the teleconsultation in nutrition has the focus on the State of Rio de Janeiro (35\% of the requests) there is a great demand from other states, especially Minas Gerais (35\%) and São Paulo (12\%). Conclusion: The results may suggest that the practice of teleconsultation in Nutrition is not yet incorporated into the routine of health primary care. Strategies are needed to strengthen the bond between health professionals and Telenutrition-RJ team, helping them to make the better use of the tool.

Keywords: Telehealth, Telenutrition, Health Primary Care, Teleconsultation

1.- Doutorado em Saúde Coletiva - Instituto de Medicina Social/UERJ - Rio de Janeiro, RJ - Brasil; 2. Mestrado em Nutrição - Instituto de Nutrição/UFRJ - Rio de Janeiro, RJ - Brasil; 3. Graduanda em Nutrição - Instituto de Nutrição/UERJ - Rio de Janeiro, RJ - Brasil 


\section{Introdução e Objetivos}

A Organização Mundial de Saúde (OMS) em 1988 já defendia o uso da tecnologia para disseminar a informação na área biomédica, melhorando assim, a qualidade prestada pelos serviços de saúde' ${ }^{1}$. Nesse contexto, destaca-se a utilização de Tecnologias de Informação e Comunicação (TIC) na saúde, quando os atores envolvidos estão distantes um do outro.

Segundo Norris², a Telessaúde pode ser definida como uso das tecnologias de informação e comunicação para transferência de informações de dados e serviços clínicos administrativos e educacionais em saúde. Neste panorama, a telessaúde é voltada não apenas para a o auxílio às práticas em saúde, mas especialmente para a promoção e a prevenção em saúde, associada a atividades como o desenvolvimento de sistemas em saúde e educação profissional na área.

Em regiões rurais ou outros tipos de comunidades mais afastadas dos centros urbanos, os recursos de TIC relacionados à saúde podem propiciar a melhoria da qualidade da assistência prestada, evitando o deslocamento de profissionais para se qualificar e dessa forma reduzindo os custos com a capacitação destes.

No Brasil e em outros países da América Latina, iniciativas desenvolvidas por governos locais, universidades e outras instituições, vêm sendo experimentadas, desencadeando importantes processos de construção de políticas públicas e de sistematização de conhecimentos na área de formação e informação em saúde.

Nessa perspectiva, o Programa Telessaúde Brasil Redes, implantado em 2007, é uma ação nacional que busca melhorar a qualidade do atendimento e da atenção básica no Sistema Único da Saúde (SUS), integrando ensino e serviço por meio de ferramentas de tecnologias da informação, que oferecem condições para promover a Teleassistência e a Teleducação.
Entre as modalidades contempladas dentro da Teleassistência, destaca-se a teleconsultoria que consiste em uma troca de informações entre profissionais da saúde remotos e locais para se discutir um caso clínico, ações em saúde ou questões relativas ao processo de trabalho ou obter uma segunda opinião, quando há necessidade de uma expertise inexistente no local, podendo ser síncrona ou assíncrona ${ }^{3}$.

Segundo a Portaria GM/MS 2.546/11, a teleconsultoria pode ser assíncrona, quando o profissional solicitante recebe a resposta por meio de mensagens offline; ou síncrona, quando é realizada uma conversa online (webconferência pré-agendada) para discussão do problema ${ }^{4}$.

As teleconsultorias assíncronas envolvem questões gerais da Atenção Primária à Saúde (APS). Podem envolver o indivíduo, a família ou comunidade, ou mesmo o processo de trabalho das equipes. Já as teleconsultorias síncronas tratam de casos clínicos ou questões de saúde mais complexas e interdisciplinares, e por isso a necessidade de ocorrer em tempo real para discussão.

Todas as atividades de teleconsultoria para APS são de apoio assistencial com caráter educacional; portanto, têm o objetivo de ampliar a capacidade resolutiva de quem as solicita. Além disso, devem ser baseadas na melhor evidência científica disponível, adaptada para as realidades locais e seguindo os princípios do Sistema Único de Saúde (SUS) e da APS 5 .

Caso uma teleconsultoria assíncrona contenha um assunto importante e replicável em outros contextos e lugares, ela pode se transformar em uma Segunda Opinião Formativa (SOF). Segundo a Portaria GM/MS 2.546/11, a SOF é resposta sistematizada, construída com base em revisão bibliográfica, nas melhores evidências científicas e clínicas e no papel ordenador da atenção básica à saúde, a perguntas originadas das teleconsultorias, e selecionadas a partir de critérios de relevância e pertinência em relação às diretrizes do SUS. 
Assim, ela é publicada com sigilo sobre o solicitante e teleconsultor na Biblioteca Virtual em Saúde (BVS) para consulta por todos 4 . Sempre é importante, antes de solicitar uma teleconsultoria, checar se existe dúvida semelhante já publicada na BVS.

O objetivo do presente estudo é relatar a experiência e apontar as potencialidades das atividades de teleconsultoria realizadas pelo núcleo de Telenutrição-RJ.

\section{Materiais e Métodos}

Trata-se de um relato de experiência tendo como fonte de dados relatórios administrativos gerados pelo sistema Moodle relacionados às atividades de Teleconsultoria realizadas pelo núcleo de Telenutrição-RJ.

\section{Resultados e Discussão}

O Telenutrição-RJ iniciou suas atividades em 2009, sendo responsável pelos conteúdos de alimentação e nutrição. É sabido que a nutrição, destacando a relação do ser humano com o alimento, é de fundamental importância na prevenção, manutenção e equilíbrio da saúde. A partir de 2012, entre as atividades do Telenutrição-RJ, associaram-se as teleconsultorias assíncronas, com um tempo máximo de resposta de 48 horas $^{6}$.

\section{Fluxo das Teleconsultorias em Nutrição}

O processo da teleconsultoria se inicia a partir da demanda do profissional solicitante motivado por suas necessidades cotidianas. A solicitação será avaliada pelo teleconsultor, que poderá requerer informações adicionais para melhor entendimento da dúvida do profissional de saúde. Assuntos muito amplos (com pouco foco) receberão respostas mais superficiais, situando a dúvida em geral, enquanto solicitações com foco bem definido receberão respostas mais direcionadas e detalhadas.

O consultor tem então 48 horas para atender a demanda e elaborar a resposta. Podem ser anexados até dois documentos utilizados para a elaboração da resposta ou que a complementem. Como exemplos de documentos, têm-se artigos científicos, diretrizes clínicas etc. Após a visualização da resposta, o profissional solicitante pode reabrir a consulta com novos questionamentos, caso seja necessário. E, novamente, sua demanda deve ser atendida em até 48 horas. Com a demanda atendida, finaliza-se o processo da teleconsultoria (Figura 1).

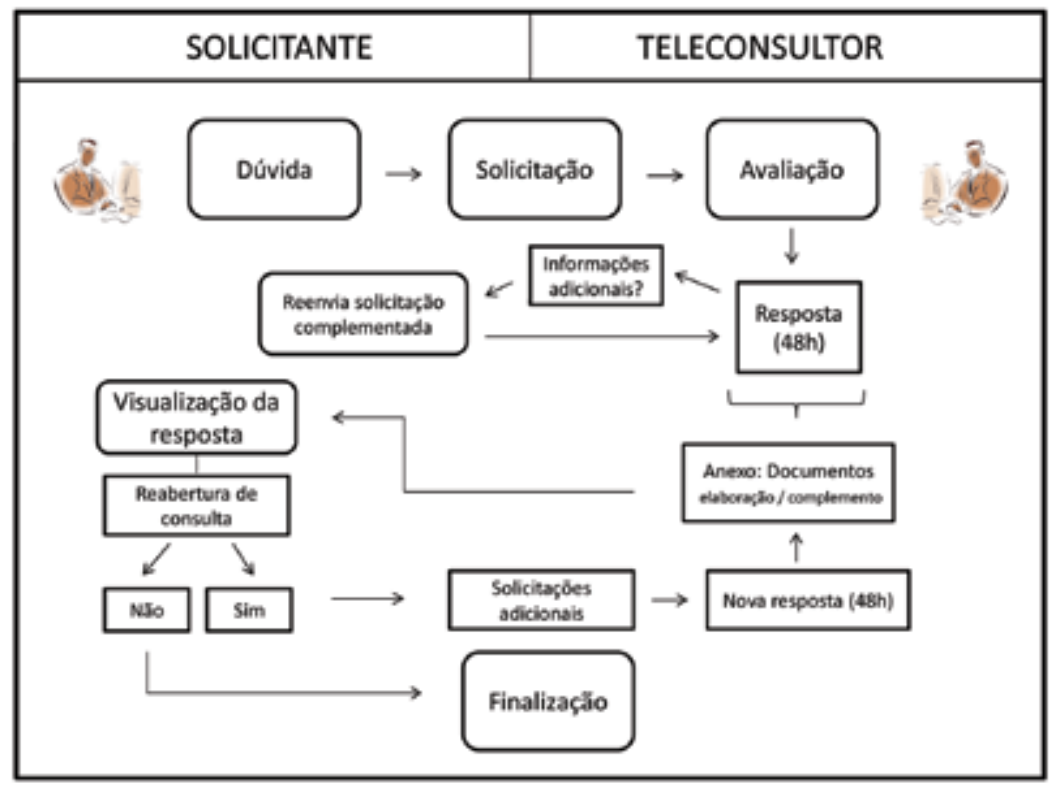

Figura 1:

Fluxo das

Teleconsultorias

realizadas pelo

Telenutrição 


\section{O perfil das teleconsultorias assíncronas realizadas pelo Telenutrição}

De abril de 2012 a setembro de 2013, foram inseridas 17 teleconsultorias no sistema do Telenutrição-RJ, oito em 2012 e nove em 2013. As solicitações foram feitas prioritariamente por nutricionistas (70\%), seguido por médicos (12\%), enfermeiro (6\%), fonoaudiólogo (6\%) e agente comunitário de saúde (6\%). Em relação à origem das solicitações, destaca-se que, apesar de o foco ser o Estado do Rio de Janeiro (seis solicitações), observa-se uma grande demanda de outros estados, principalmente de Minas Gerais (seis solicitações) e São Paulo (duas solicitações), conforme demonstrado no gráfico 1.

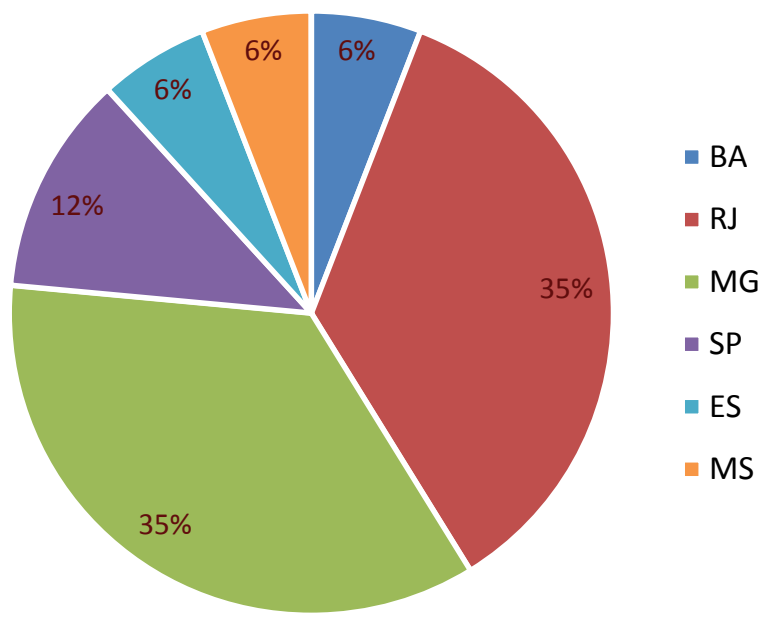

Gráfico 1: Origem das Teleconsultorias solicitadas, 2012-2013.

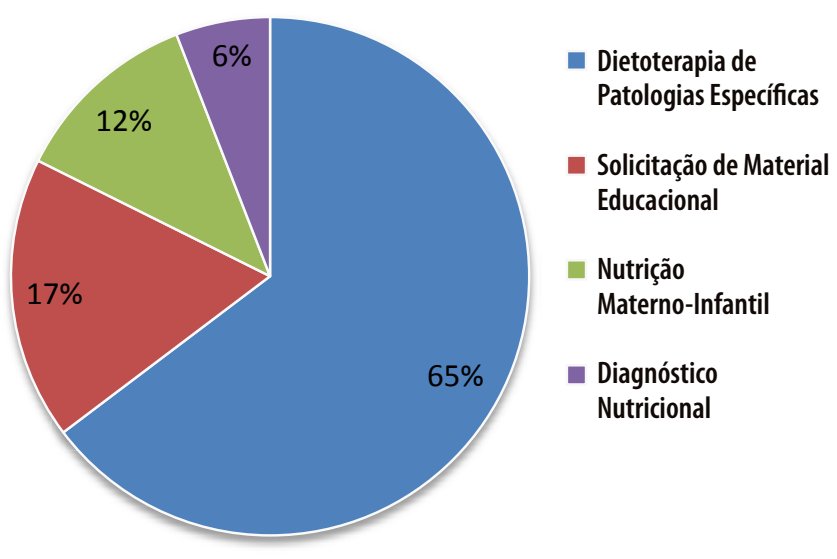

Gráfico 2: Classificação das Solicitações das Teleconsultorias, 2012-2013.
As dúvidas solicitadas apresentaram uma diversidade de assuntos, conforme o gráfico 2, com uma grande demanda relacionada à dietoterapia de patologias específicas (11 solicitações), solicitação de material educacional (3 solicitações), nutrição materno-infantil (duas solicitações) e diagnóstico nutricional (1 solicitação).

Como o número de solicitações é baixo, há uma diversidade de assuntos, porém destaca-se que houve repetição de duas dúvidas relacionadas ao tratamento dietoterápico, uma repetição sobre a Doença de Crohn, e outra sobre a desnutrição em idosos.

A teleconsultoria é uma nova forma de trabalho, porém ainda pouco inserida no cotidiano dos profissionais da atenção primária de saúde. Alguns autores discutem que fatores organizacionais são fundamentais para a adoção e incorporação dessa ferramenta ${ }^{7,8}$. São necessárias novas rotinas, exigindo um planejamento e reestruturação para que a teleconsultoria seja incorporada na prática diária. Portanto, envolve além dos profissionais de saúde, o gestor de saúde dos municípios.

O núcleo Telenutrição-RJ é pioneiro na área, sendo o único no Brasil. A baixa demanda pode ser em parte justificada pela baixa inserção do profissional nutricionista na APS. Com o objetivo de ampliar o escopo das ações de Atenção Básica, a partir de 2008 foram criados os Núcleos de Apoio à Saúde da Família (NASF), que se caracterizam pela constituição em equipes multiprofissionais, pertencentes à ESF, atuantes no âmbito da saúde e assistência social, sendo o nutricionista um dos profissionais que pode fazer parte delas ${ }^{9}$. A partir da implantação dos NASF, observa-se que a inserção profissional do nutricionista na atenção básica vem se ampliando principalmente em grandes centros urbanos $^{10}$. Espera-se, assim, que a demanda por Teleconsultorias em Nutrição também seja ampliada.

Algumas estratégias utilizadas por outros núcleos de Teleconsultorias, principalmente da Telemedicina podem ser adotadas a fim de se avaliar a ferramenta, 
como exemplo, a avaliação da Teleconsultoria prestada, a satisfação com o serviço e a facilidade do sistema, gerando subsídios para a melhoria das ações.

Outras ações também devem ser mencionadas, como a capacitação dos gestores da saúde em Telessaúde, demonstrando seus benefícios e a criação de condições para o uso de tecnologias de informação e comunicação na rotina dos serviços de saúde. A incorporação do uso da Teleducação e Teleassistência, destacando o potencial de suporte aos profissionais de saúde, nas disciplinas da graduação é outra ação que pode aumentar o conhecimento dos benefícios da ferramenta com o intuito de facilitar sua incorporação na rotina de trabalho das gerações futuras.

\section{Conclusão}

A disponibilização de ferramentas de informação que atendam às necessidades dos profissionais nutricionistas é fundamental. Os primeiros resultados que avaliaram as Teleconsultorias em Nutrição - RJ evidenciam uma baixa incorporação por profissionais de saúde em seu cotidiano de trabalho. Estratégias que promovam o vínculo entre profissionais e equipe do Telenutrição, treinamento constante e atendimento às necessidades locais podem auxiliar na melhor utilização da ferramenta.

\section{Referências}

1. World Health Organization (1988) Learning together to work together for health. Report of a WHO Study Group on Multiprofessional Educaton of Health Personnel and the Team Approach,World Health Organisation, Geneva, 796.

2. Norris AC. Essentials of telemedicine and Telecare. Baffn Lane - England: John Wiley \& Son, 2002

3. Nerlich M, Balas EA, Schall T, Stieglitz SP, Filzmaier R, Asbach P et al. Teleconsultation Practice Guidelines: Report from G8 Global Health Applications Subproject 4. Telemed J E Health. 2002;8(4):411-8.

4. Brasil. Ministério da Saúde. Portaria GM/MS 2.546, de 27 de outubro de 2011. Redefine e amplia o Programa Telessaude Brasil, que passa a ser denominado Programa Nacional Telessaude Brasil Redes (Telessaude Brasil Redes). Diário Oficial da União, Brasília-DF, Seção 1, 28 fev. 2011.

5. Brasil. Ministério da Saúde. Manual de Telessaúde para Atenção Básica / Atenção Primária à Saúde / Ministério da Saúde, Universidade Federal do Rio Grande do Sul. Brasília: Ministério da Saúde, 2012.

6. Valle J, Cury MTF. Telenutrição: Mudando paradigmas na Educação em Saúde. Rev HUPE. 2012;11:40-4

7. Durrani H, Khoja S. A systematic review of the use of telehealth in Asian countries. J Telemed Telecare. 2009;15:175-81.

8. Moehr JR, Schaafsma J, Anglin C, Pantazi SV, Grimm NA, Anglin S. Success factors for telehealth - a case study. Int J Med Inform. 2006;75(10-11):755-63.

9. Brasil. Portaria no 154 de 24 de janeiro de 2008. Cria os Núcleos de Apoio à Saúde da Família (NASF). Diário Oficial da União 2008; 24 jan.

10. Cervato-Mancuso AM, Tonacio LV, Silva, ER, Vieira, VL. A atuação do nutricionista na Atenção Básica à Saúde em um grande centro urbano. Ciência e Saúde Coletiva. 2012;17(12), 3289-300. 